\title{
SUBJECTIVE PROBABILITIES NEED NOT BE SHARP
}

(forthcoming in Erkenntnis)

\section{JAKE CHANDLER*}

\begin{abstract}
It is well known that classical, aka 'sharp', Bayesian decision theory, which models belief states as single probability functions, faces a number of serious difficulties with respect to its handling of agnosticism. These difficulties have led to the increasing popularity of so-called 'imprecise' models of decision-making, which represent belief states as sets of probability functions. In a recent paper, however, Adam Elga has argued in favour of a putative normative principle of sequential choice that he claims to be borne out by the sharp model but not by any promising incarnation of its imprecise counterpart. After first pointing out that Elga has fallen short of establishing that his principle is indeed uniquely borne out by the sharp model, I cast aspersions on its plausibility. I show that a slight weakening of the principle is satisfied by at least one, but interestingly not all, varieties of the imprecise model and point out that Elga has failed to motivate his stronger commitment.
\end{abstract}

Keywords Elga - imprecise probabilities - sequential choice

*Address for correspondence: Center for Logic and Analytic Philosophy, HIW, KU Leuven, Kardinaal Mercierplein 2, 3000 Leuven, Belgium. Email: jacob.chandler@hiw. kuleuven. be 


\section{Introduction}

Championed by the likes of Isaac Levi (1980), Peter Walley (1991) and many others, so-called 'imprecise' probabilistic models of rational decison-making have become increasingly influential in the past few decades, permeating disciplines ranging from philosophy to statistics, through engineering, artificial intelligence and economics. ${ }^{1}$ These models relax the rationality requirements of standard 'sharp' Bayesian decision theory by representing the credal states of rational agents, not by a unique probabilistically coherent credence function, but by a set of such functions and opting for some generalisation or other of the standard Bayesian decision rule. It is argued that this move notably yields a more adequate representation of suspension of judgment, a state of mind that the sharp model has serious difficulties in handling. ${ }^{2}$

In an interesting and provocative recent article, however, Adam Elga (2010) has argued that this liberalisation of Bayesian orthodoxy is a step in the wrong direction. According to him, the imprecise view falters in its handling of sequential choice problems, licensing behaviours that are intuitively rationally reprehensible. More precisely, he argues for the following pair of claims:

(i) The normative claim: Any satisfactory model of rational decision making should vindicate a certain conditional principle of rational choice-call it '(P)'that states that, given certain very weak conditions, a rational agent will refrain from making a particular kind of sequence of decisions.

(ii) The descriptive claim: (P) is borne out by the sharp model but not by any promising incarnation of its imprecise counterpart.

Elga's contention, if correct, would be of considerable significance. It would, in particular, make for extremely disturbing news for those who hold that the aforementioned difficulties facing the sharp view are insuperable. (Elga himself, one should point out, is rather more sanguine on that front. I shall not, however, be discussing his alleged grounds for optimism here.)

However, as I shall argue, Elga's does not offer a convincing case against the imprecise view. After briefly noting that Elga has not successfully established the truth of his descriptive claim (ii), I shall argue that his normative claim (i) is equally unsupported. More specifically, I will point out that he provides us with no motivation to commit ourselves to $(\mathrm{P})$ rather than to merely a slightly weaker cognate principle $\left(\mathrm{P}^{-}\right)$, obtained by a mild strengthening of $(\mathrm{P})$ 's antecedent. This is a significant shortcoming, since it turns out that $\left(\mathrm{P}^{-}\right)$is satisfied by at least one well known variety of the imprecise model: without providing us with a clear reason to

\footnotetext{
${ }^{1}$ For a survey of the imprecise approach, see for instance Miranda (2008) or Troffaes (2007).

${ }^{2}$ I shall briefly review some of these difficulties later on in the paper, for those readers who may not be familiar with them.
} 
plump for the stronger principle, Elga does not have a case against the imprecise view. Having said this, we shall see that even $\left(\mathrm{P}^{-}\right)$is not entirely toothless: it remains violated by those imprecise models that lie at the very 'liberal' end of the decision-theoretic spectrum. This last observation has a potential bearing on the internal dispute between competing varieties of the imprecise view.

The structure of the article is as follows. Section 2 provides a brief overview of the sharp view and a reminder of some of the pertinent difficulties that it faces. Section 3 introduces some of the principal variants of its imprecise counterpart, noting that these circumvent the aforementioned issues. Section 4 presents Elga's objection. Sections 5 and 6 discuss Elga descriptive and normative claims, respectively, and present the key technical contribution of the paper, namely the proof of the aforementioned claim regarding $\left(\mathrm{P}^{-}\right)$. Section 7 briefly concludes. Proofs of those formal claims that are not central to the discussion are provided in the Appendix.

\section{The sharp view and some associated difficulties}

There exist a fair number of variants of the 'sharp' model of rational choice in the literature. For the purposes of this paper, however, it will suffice to outline a version of Richard Jeffrey's so-called evidential incarnation of the framework (Jeffrey 1983). The subsequent discussion carries over to cognate accounts.

As is customary, we start off with a set of propositions, modeled as the powerset $\wp(W)$ of the set $W$ of all possible worlds. This set represents a given rational agent $S$ 's 'language of thought': the set of propositions with respect to which he or she can hold various mental attitudes. These attitudes are taken to come in two varieties: doxastic-pertaining to what the agent believes-and bulativepertaining to what the agent desires. $S$ 's doxastic attitudes at time $t$ are represented by a probability function $\operatorname{Pr}_{t}$ with domain $\wp(W)$, capturing $S$ 's assessment of the respective probabilities of each proposition. This function is updated by so-called strict conditionalisation, so that the updated probability of a proposition $P$ upon learning a proposition $Q$ is given by its prior conditional probability given $Q .{ }^{3} S$ 's bulative attitudes at time $t$ are represented by a utility function $\mathrm{U}_{t}$ with domain $W$, capturing, for each world, the value that $S$ would attach to it's being the one that he or she is situated in. This valuation is then extended to each proposition $P$ by considering its expected utility, or, more precisely, the expectation $\mathrm{E}_{\mathrm{Pr}_{t}}\left(\mathrm{U}_{t} \mid P\right)$ of $\mathrm{U}_{t}$ conditional on $P$, with respect to $\mathrm{Pr}_{t}{ }^{4}$ In what follows, we shall assume that the agent's utility function is invariant across times and omit the temporal indexing of U.

At each time, $S$ is assumed to have the capacity to make true some proposition

\footnotetext{
${ }^{3}$ More precisely: for all $Q \in \wp(W)$, if $Q$ the strongest proposition learnt by $S$ between $t$ and $t+1$, then for all $P \in \wp(W), \operatorname{Pr}_{t+1}(P)$ is set equal to $\operatorname{Pr}_{t}(P \mid Q)$.

${ }^{4} \mathrm{E}_{\mathrm{Pr}_{t}}\left(\mathrm{U}_{t} \mid P\right)$ is given by $\left[\Sigma_{w \in P} \mathrm{U}_{t}(w) \operatorname{Pr}_{t}(\{w\})\right] / \operatorname{Pr}(P)$, on the assumption that $W$ is countable.
} 
(an 'act') drawn from a partition of $W$ (an 'alternative set'). A choice function $\mathrm{C}_{t}$ returns the subset of acts that are rationally permissible at time $t$, those propositions that our rational agent $S$ is liable to choose to make true at $t$, in a given alternative set (the 'choice set'). Choiceworthiness is taken to go by maximisation of the expected utility of the act. ${ }^{5}$

Notoriously, this kind of framework has faced a wide variety of criticisms, most notably regarding its use of conditional expectations in the construction of choice sets. On the one hand, advocates of so-called causal decision theory complain that this counterintuitively promotes the choice of acts that are taken to merely correlate with, rather than causally promote, desirable states of affairs. ${ }^{6}$ On the other hand, defenders of various forms of non-expected utility theory have criticized the socalled linearity in the probabilities that this feature of the model imposes. ${ }^{7}$

These worries, however, are entirely tangential to our current concerns. The objections that are of relevance in the present context pertain instead to the framework's handling of decision-making in cases of radical lack of opinionation. Since these will no doubt be familiar to many readers, I will keep the presentation succinct.

The first issue, which is arguably the thorniest, is the observation that the sharp model is at odds with a trio of plausible propositions regarding agnosticism with respect to the value of random variables. Indeed, first of all, it seems plausible that (1), for any two symmetrically-related random variables $\mathrm{X}$ and $\mathrm{Y}$, if a rational agent is agnostic with respect to the value of $\mathrm{X}$, then she is also agnostic with respect to the value of $Y$. Second, it is surely the case that (2), for any random variable X, if a rational agent is agnostic with respect to the value of $X$, then, for any two intervals $I_{1}$ and $I_{2}$ of equal length that lie within the range of $\mathrm{X}$, it is not the case that the agent is strictly more confident that the true value of X lies in $I_{1}$ than she is that it lies in $I_{2}$. Finally, clearly (3) there could exist a rational agent $S$ and a continuous random variable $\mathrm{X}$, such that $S$ is agnostic with respect to the value of $\mathrm{X}$.

Unfortunately, however, it is easy to show that the sharp view is inconsistent with the conjunction of these three intuitions. To see why, assume first that (3) is true, so that $S$ is agnostic with respect to the value of some continuous random variable X. It follows from (1) that $S$ is agnostic with respect to the value of the symmetrically-related random variable $1 / \mathrm{X}$. Now assume that (2) is true. Given the sharp view, it then follows that $S$ 's opinions with respect to the value of $\mathrm{X}$ are to be represented by a uniform probability density function over the range of $\mathrm{X}$. But as a consequence of this, $S$ 's opinions with respect to the value of $1 / \mathrm{X}$ are to be represented by a probability density function over the range of $1 / \mathrm{X}$ that is not

\footnotetext{
${ }^{5}$ In other words, where $\mathbb{A}$ denotes a partition of $W$ and $A \in \mathbb{A}, C_{t}$ satisfies:

MAx EU: $A \in \mathrm{C}_{t}(\mathbb{A})$ iff for all $B \in \mathbb{A}, \mathrm{E}_{\mathrm{Pr}_{t}}\left(\mathrm{U}_{t} \mid A\right) \geq \mathrm{E}_{\mathrm{Pr}_{t}}\left(\mathrm{U}_{t} \mid B\right)$

${ }^{6}$ See Joyce (1999, Ch. 5) or Weirich (2008) for recent surveys.

${ }^{7}$ See Machina (1987) for an influential overview.
} 
uniform. So by (2) again, $S$ is not agnostic with respect to the value of $1 / \mathrm{X}$ after all. Contradiction. ${ }^{8}$

The second difficulty, famously noted by Ellsberg (1961), is that the sharp model would appear to impose counterintuitive constraints on choice prescriptions with respect to simple decision problems involving partial agnosticism. Indeed, consider the following scenario: A subject $S$ is faced with an urn containing a number of balls, one third of which she takes to be red and two thirds of which she takes to be a mixture of yellow and black. $S$, however, suspends judgment on the ratio of yellow to black balls. She is then confronted, at time $t$, with two separate alternative sets, $\mathbb{A}=\left\{A_{1}, A_{2}\right\}$ and $\mathbb{B}=\left\{B_{1}, B_{2}\right\}$. Gamble $A_{1}$ yields a payoff of 100 utiles if the ball is red, and nothing otherwise. Gamble $A_{2}$ yields 100 utiles if the ball is yellow, and nothing otherwise. Finally, gambles $B_{1}$ and $B_{2}$ are identical to $A_{1}$ and $A_{2}$ respectively, save that 100 utiles are also won in case the ball is black. It is easy to show that, according to the sharp model, if $\mathrm{C}_{t}(\mathbb{A})=\left\{A_{i}\right\}$ then $\mathrm{C}_{t}(\mathbb{B})=\left\{B_{i}\right\}$. However, it turns out that the modal intuition is that $\mathrm{C}_{t}(\mathbb{A})=\left\{A_{1}\right\}$ and $\mathrm{C}_{t}(\mathbb{B})=\left\{B_{2}\right\}^{9}$

\section{The imprecise view and some associated selling points}

In the imprecise framework, $S$ 's doxastic attitudes at time $t$ are modeled, not by a single probability function, but by a whole set $\Gamma_{t}$ of such functions, sometimes known as a 'credal set'. ${ }^{10}$ Belief dynamics and constraints on choice sets are correspondingly generalised, although there does remain a certain lack of consensus regarding the appropriate way to go about this. Regarding dynamics, credal sets are standardly updated by individually conditionalising each of the functions that they contain. ${ }^{11}$ Regarding the construction of choice sets, a number of alternatives have been offered in the literature. The principal suggestions are the following:

$\Gamma$-Maximin: An act $A$ is rationally permissible if and only if, for any alternative act $B$, the lowest expected utility of $A$ is at least as great as the lowest expected utility of $B$.

$\Gamma$-Maximax: An act $A$ is rationally permissible if and only if, for any alternative act $B$, the highest expected utility of $A$ is at least as great as the highest

\footnotetext{
${ }^{8}$ This is one way of presenting the core content of the so-called paradoxes of indifference. See van Fraassen (1989, Ch. 12) for an excellent presentation of these, and Shackle (2007) for a critical treatment of some of the replies made on behalf of the sharp view.

${ }^{9}$ See Camerer \& Weber (1992) for a survey of some of the experimental data.

${ }^{10}$ This set is required by some, most notoriously Levi (1980), to be convex. See Jeffrey (1987), however, for convincing reasons to hold that this constraint is not in order.

${ }^{11}$ Note however that this popular suggestion has attracted a certain degree of controversy, since it countenances a phenomenon known as 'probabilistic dilation', which some, such as White (2010), have judged to be counterintuitive. See Joyce (2010) for a defense of the claim that such controversy is unwarranted. The first in-depth formal discussion of dilation was due to Seidenfeld \& Wasserman (1993).
} 
expected utility of $B$.

E-Admissibility: An act $A$ is permissible if and only if there is a probability function $\operatorname{Pr}$ in the agent's credal set such that, according to $\operatorname{Pr}$, for any alternative act $B$, the expected utility of $A$ is at least as great as that of $B$.

Maximality: An act $A$ is permissible if and only if, for any alternative act $B$, there exists a probability function Pr in the agent's credal set according to which the expected utility of $A$ is at least as great as that of $B$.

Interval Dominance: An act $A$ is rationally permissible if and only if, for any alternative act $B$, the highest expected utility of $A$ is at least as great as lowest expected utility of $B .^{12}$

These constraints have been partially ordered by decreasing stringency, an ordering that can be conveniently exploited in the proof of various results; see Figure 1, reproduced from Troffaes (2007).

It turns out that the aforementioned difficulties faced by the sharp view are easily circumvented in this more general representational framework. Regarding the first issue, suspension of judgment with respect to the value of a continuous random variable $\mathrm{X}$ is standardly modeled by having every probability density function whose domain is the range of $\mathrm{X}$ be represented in $\Gamma_{t}$. It is also equally standard to offer what one might call a 'supervaluationist' construal of possession of strictly greater confidence in $P$ than in $Q$, so that the latter is represented by its being the case that $\forall \operatorname{Pr} \in \Gamma_{t}, \operatorname{Pr}(P)>\operatorname{Pr}(Q)$. Given these two modeling choices, the imprecise model is not only consistent with, but indeed entails, the conjunction of the aforementioned trio of propositions. As for the second issue, i.e. Ellsberg's urn, it is easily verified that the adoption of a suitable decision rule, such as Г-MAximin, enables one to recover the desired modal intuition.

\section{Elga's argument against the imprecise view}

So what is there not to like about the imprecise view? Elga claims that it faces a hitherto unnoticed difficulty. He asks us to consider a a pair of decision problems faced in sequence. At time 1, the agent faces a choice between $A_{1}$, accepting a

\footnotetext{
${ }^{12}$ More compactly, where $A \in \mathbb{A}$ :

$\Gamma$-Maximin: $A \in \mathrm{C}_{t}(\mathbb{A})$ iff $\forall B \in \mathbb{A}, \min \left\{\mathrm{E}_{\operatorname{Pr}}(\mathrm{U} \mid A): \operatorname{Pr} \in \Gamma_{t}\right\} \geq \min \left\{\mathrm{E}_{\mathrm{Pr}}(\mathrm{U} \mid B): \operatorname{Pr} \in \Gamma_{t}\right\}$

$\Gamma$-Maximax: $A \in \mathrm{C}_{t}(\mathbb{A})$ iff $\forall B \in \mathbb{A}, \max \left\{\mathrm{E}_{\operatorname{Pr}}(\mathrm{U} \mid A): \operatorname{Pr} \in \Gamma_{t}\right\} \geq \max \left\{\mathrm{E}_{\operatorname{Pr}}(\mathrm{U} \mid B): \operatorname{Pr} \in \Gamma_{t}\right\}$

E-Admissibility: $A \in \mathrm{C}_{t}(\mathbb{A})$ iff $\exists \operatorname{Pr} \in \Gamma_{t}, \forall B \in \mathbb{A}, \mathrm{E}_{\mathrm{Pr}}(\mathrm{U} \mid A) \geq \mathrm{E}_{\mathrm{Pr}}(\mathrm{U} \mid B)$

Maximality: $A \in \mathrm{C}_{t}(\mathbb{A})$ iff $\forall B \in \mathbb{A}, \exists \operatorname{Pr} \in \Gamma_{t}, \mathrm{E}_{\operatorname{Pr}}(\mathrm{U} \mid A) \geq \mathrm{E}_{\operatorname{Pr}}(\mathrm{U} \mid B)$.

Interval Dominance: $A \in \mathrm{C}_{t}(\mathbb{A})$ iff $\forall B \in \mathbb{A}, \max \left\{\mathrm{E}_{\mathrm{Pr}}(\mathrm{U} \mid A): \operatorname{Pr} \in \Gamma_{t}\right\} \geq \min \left\{\mathrm{E}_{\mathrm{Pr}}(\mathrm{U} \mid B)\right.$ : $\left.\operatorname{Pr} \in \Gamma_{t}\right\}$
} 


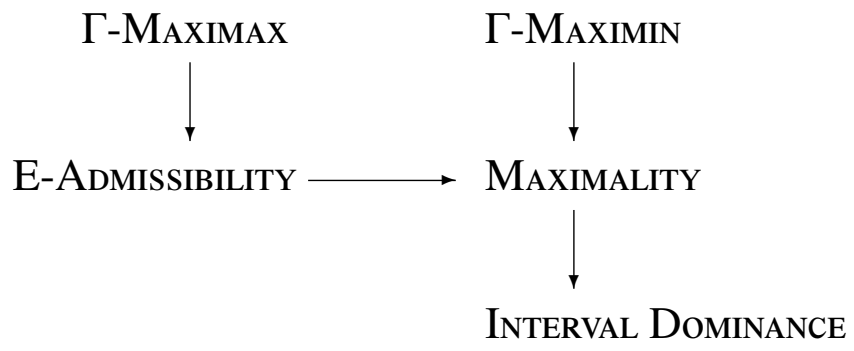

Figure 1: Partial ordering of common imprecise decision rules by degree of stringency.

bet that pays out -10 utiles in case environmental state $H$ obtains and 15 utiles in case $H$ is false and $A_{2}$, the status quo, that pays out 0 utiles irrespective of whether $H$ obtains. At time 2 , the agent faces a choice between between $B_{1}$, accepting a bet that pays 15 utiles in case $H$ is true and -10 utiles in case $H$ is not, and $B_{2}$, the status quo, that pays out 0 utiles irrespective of whether $H$ is the case. It is assumed $S$ considers states and acts be independent. The corresponding decision tree is depicted in Figure 2 below.

As Elga notes, choosing both $A_{1}$ and $B_{1}$ secures a sure payoff of 5 utiles, a prospect that is clearly more desirable than the one resulting from choosing both $A_{2}$ and $B_{2}$ instead, i.e. making the decision to decline both gambles and run with the status quo. With this observation in hand, Elga then moves on to make the following normative claim:

(P) A rational agent will either choose $A_{1}$ or choose $B_{1}$, given:

Full disclosure (FD): For all functions in $\Gamma_{1}$, the probability assigned to the agent's choosing one of either $B_{1}$ or $B_{2}$ at time 2 is 1 .

No Change in Belief in $H(N C)$ : There is a function $\operatorname{Pr}$ in $\Gamma_{1}$ such that $\operatorname{Pr}(H)=r$ if and only if there is such a function in $\Gamma_{2} \cdot{ }^{13}$

\footnotetext{
${ }^{13}$ This is my 'precisification' of the principle, as originally stated. Elga's own words are:

Any perfectly rational agent who is sequentially offered bets $\left[A_{1}\right]$ and $\left[B_{1}\right]$ in the above circumstances (full disclosure in advance about the whole setup, no change of belief in $H$ during the whole process,... ) will accept at least one of the bets. (ibid, p. 4)
} 


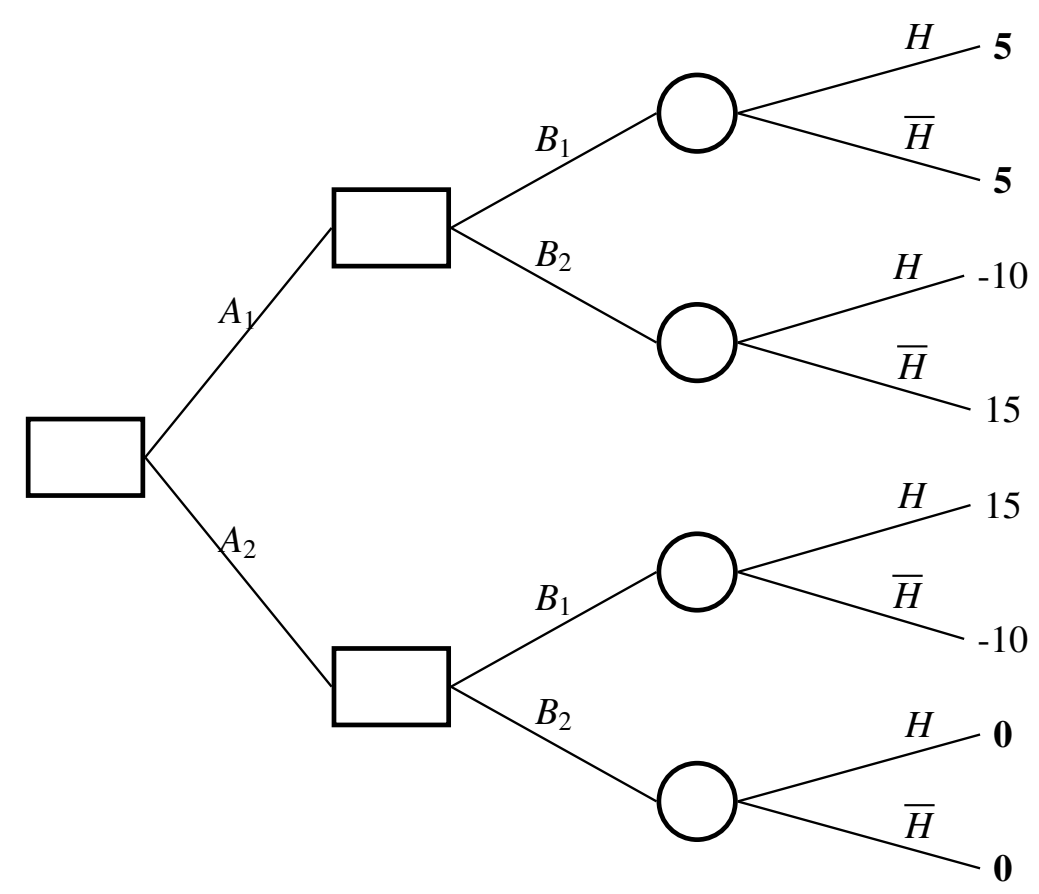

Figure 2: Elga's decision problem.

The problem however, according to Elga, is that whilst $(\mathrm{P})$ is satisfied by the standard Bayesian model, it is not borne out by any of the most popular imprecise models of rational choice: 'Those who think rational agents always have sharp probability functions', he tells us, 'can easily accommodate [(P)]. [But those who do not] cannot accommodate it at all.' (ibid, p. 4). (Call this the 'descriptive claim'.) On the basis of these considerations, he then concludes that 'subjective probabilities should be sharp'.

\section{Elga's descriptive claim}

Elga's descriptive claim should be motivated by the following: a proof that, on the one hand, any imprecise model satisfying some minimal set of desiderata violates $(\mathrm{P})$, but, on the other, the sharp model does not. Such a demonstration, however, is not provided. In particular, the only proof given in relation to the imprecise view pertains to E-AdmissibiLity. But this is one of the more permissive rules considered in Section 3, and as such constitutes a relatively soft target. No grounds, for instance, are provided to even hold that the most stringent rules that we have considered here, namely $\Gamma$-MAXIMIN and $\Gamma$-MAXIMAX, violate the said principle.

It is also worth noting that, to the extent that a formal proof of E-ADMISsiBILITY's violation of $(\mathrm{P})$ can be reconstructed from Elga's comments, the derivation of the

He does not elaborate on the precise content of either 'full disclosure in advance about the whole setup' or 'no change of belief in $H$ during the whole process'. 
result is problematic. Indeed, his line of reasoning would appear to be the following:

Let $\Gamma_{1}$ be such that the range of probabilities for $H$ span the interval $[0.2,0.8]$. It then follows from (NC) that there exists $\operatorname{Pr}_{1} \in \Gamma_{1}$ such that $\operatorname{Pr}_{1}(H)=0.8$ and $\operatorname{Pr}_{2} \in \Gamma_{2}$ such that $\operatorname{Pr}_{2}(H)=0.2$. The conditional expected utilities of $A_{1}$ and $A_{2}$, given $H$ and given $\bar{H}$, with respect to $\operatorname{Pr}_{1}$ are given as follows:

$$
\begin{aligned}
& \mathrm{E}_{\mathrm{Pr}_{1}}\left(\mathrm{U} \mid A_{1} \cap H\right)=-10 \\
& \mathrm{E}_{\mathrm{Pr}_{1}}\left(\mathrm{U} \mid A_{1} \cap \bar{H}\right)=15 \\
& \mathrm{E}_{\mathrm{Pr}_{1}}\left(\mathrm{U} \mid A_{2} \cap H\right)=\mathrm{E}_{\mathrm{Pr}_{1}}\left(\mathrm{U} \mid A_{2} \cap \bar{H}\right)=0
\end{aligned}
$$

From this, assuming probabilistic independence of acts and states with respect to $\operatorname{Pr}_{1}$, we obtain the following inequality between the unconditional expected utilities of these acts, with respect to $\operatorname{Pr}_{1}$ : $\mathrm{E}_{\mathrm{Pr}_{1}}\left(\mathrm{U} \mid A_{1}\right)=-5<$ $\mathrm{E}_{\mathrm{Pr}_{1}}\left(\mathrm{U} \mid A_{2}\right)=0$. It then follows by E-AdmissibiLITY that $A_{2} \in \mathrm{C}_{1}\left(\left\{A_{1}, A_{2}\right\}\right)$. Now the conditional expected utilities of $B_{1}$ and $B_{2}$, given $H$ and given $\bar{H}$, with respect to $\operatorname{Pr}_{2}$ are given as follows:

$$
\begin{aligned}
& \mathrm{E}_{\mathrm{Pr}_{2}}\left(\mathrm{U} \mid B_{1} \cap H\right)=15 \\
& \mathrm{E}_{\mathrm{Pr}_{2}}\left(\mathrm{U} \mid B_{1} \cap \bar{H}\right)=-10 \\
& \mathrm{E}_{\mathrm{Pr}_{2}}\left(\mathrm{U} \mid B_{2} \cap H\right)=\mathrm{E}_{\mathrm{Pr}_{2}}\left(\mathrm{U} \mid B_{2} \cap \bar{H}\right)=0
\end{aligned}
$$

From this, assuming probabilistic independence of acts and states with respect to $\operatorname{Pr}_{2}$, we obtain the following inequality between the the unconditional expected utilities of these acts, with respect to $\operatorname{Pr}_{1}$ : $\mathrm{E}_{\mathrm{Pr}_{2}}\left(\mathrm{U} \mid B_{1}\right)=-5<$ $\mathrm{E}_{\mathrm{Pr}_{2}}\left(\mathrm{U} \mid B_{2}\right)=0$. It then follows by E-AdmissibiLITY that $B_{2} \in \mathrm{C}_{1}\left(\left\{B_{1}, B_{2}\right\}\right)$.

But of course statements (1) and (2) above needn't hold true. Indeed, the total payoff eventually received, conditional upon making a certain choice at a certain time $i$, depends not just on whether or not $H$ obtains, but also on the nature of the choice that was made or will be made at the other time $j$. In other words, the calculations offered in (1) and (2) should have taken into account the probabilityweighted payoffs of the agent's possible choices at times 2 and 1 respectively. ${ }^{14}$ So for instance, given (FD), $\mathrm{E}_{\mathrm{Pr}_{2}}\left(\mathrm{U} \mid A_{1} \cap H\right)$ should have instead been calculated

\footnotetext{
${ }^{14} \mathrm{An}$ anonymous referee has raised concerns regarding the stringency of the requirement, implicit in the decision theoretic framework employed here, that an agent factor into her calculations of the desirability of a given choice the repercussions that this choice may have on her potential future decisions. This is indeed non-trivial constraint. But then again, the same could be said of many of the other constraints that decision theory standardly imposes (e.g. probabilistic coherence). The nature of the 'contrary-to-duty' obligations incurred when these more demanding requirement are violated remains largely uncharted territory. Furthermore, in the present case of interest, the agent supposedly receives 'full disclosure in advance about the whole setup', so that she is explicitly made aware at time 1 of the impending decision to be made at time 2. It is hard to imagine that, under such conditions, even a very liberal decision theory would licence the relevant kind of short-sightedness.
} 
using the following formula:

$$
\operatorname{Pr}_{1}\left(B_{2} \mid A_{1} \cap H\right)(-10)+\operatorname{Pr}_{1}\left(B_{1} \mid A_{1} \cap H\right)(5)
$$

As things stand, the proof of even this limited part of the claim requires further work. ${ }^{15}$ Elga has not done enough to substantiate his allegation that the truth of (P) would leave the imprecise view in a comparative state of theoretical bankruptcy. ${ }^{16}$

\section{Elga's normative claim}

It is not, however, just the legitimacy of the descriptive claim that remains to be established: its normative complement is no closer to having been secured. Indeed Elga quite explicitly concedes that he is not in a position to offer any independent considerations in favour of the latter:

One might insist that rejecting both bets [given (FD) and (NC)] is consistent with perfect rationality. I do not find this plausible, but have no additional arguments. I can only ask you to vividly imagine a case in which an agent rejects both bets $\left[A_{1}\right]$ and $\left[B_{1}\right] \ldots$ I invite you to agree that this agent has exhibited a departure from perfect rationality. (ibid, p. 6)

But to unreflectively accept Elga's invitation would be somewhat irresponsible, given what he claims to be at stake. Indeed, granting his descriptive claim, the very fate of the imprecise view would hang in the balance. A certain degree of caution should be taken here.

With this in mind, consider now the following question: Even if one were inclined to concede that something in the vicinity of $(\mathrm{P})$ is intuitively compelling, so that under conditions of a certain particular kind, a rational agent will either choose $A_{1}$ or choose $B_{1}$, why be so certain that the specific conditions offered by Elga jointly suffice?

One might be tempted to dismiss the worry by claiming that $(\mathrm{P})$ is simply derivable from an already familiar and uncontroversial decision-theoretic principle. As was noted above, in Section 4, whether or not $H$ turns out to be true, choosing both individually 'safe' prospects leaves $S$ strictly worse off, from her perspective, than choosing both individually 'risky' ones. In decision-theoretic parlance, the intersection of the risky prospects 'strictly dominates' the intersection of the safe ones.

\footnotetext{
${ }^{15}$ It turns out that this particular subclaim is in fact true. Indeed, further down, I provide a proof that E-AdmissiBiLity violates a principle that is strictly weaker that $(\mathrm{P})$.

${ }^{16}$ One should point out that, although Elga does not offer a proof that $(\mathrm{P})$ is indeed satisfied by the sharp model, this result can be derived, and without relying on the problematic calculations of expected utility that were flagged out above. The proof, kindly supplied by an anonymous referee, can be found in the Appendix.
} 
So, the thought might go, is it not just the case that $(\mathrm{P})$ is a mere consequence of the following widely-endorsed principle of StRIct Dominance?

Strict Dominance: At no time $t$ will a rational agent $S$ will choose, from a given alternative set $\mathbb{A}$, an act that is strictly dominated by another, where $A$ is strictly dominated by $B$ iff for some partition $\mathbb{E}$ of propositions that $S$ regards to be independent of $\mathbb{A}$, for all $E \in \mathbb{E}, \mathrm{E}_{\mathrm{Pr}_{t}}\left(\mathrm{U}_{t} \mid B \cap E\right)>\mathrm{E}_{\mathrm{Pr}_{t}}\left(\mathrm{U}_{t} \mid A \cap E\right) .{ }^{17}$

Unfortunately not, and it takes but a moment's reflection to realise why. Indeed, whilst it is of course true that $A_{2} \cap B_{2}$ strictly dominates $A_{1} \cap B_{1}$, it is assumed in the situation considered that at no point is the agent in a position to choose between these two options. ${ }^{18}$ Indeed, at time 1 , she chooses between $\left(A_{1} \cap B_{1}\right) \cup\left(A_{1} \cap B_{2}\right)$ and $\left(A_{2} \cap B_{1}\right) \cup\left(A_{2} \cap B_{2}\right)$. At time 2, depending on her choice at time 1 , she either (i) chooses between $A_{1} \cap B_{1}$ and $A_{1} \cap B_{2}$, or (ii) chooses between $A_{2} \cap B_{1}$ and $A_{2} \cap B_{2}$. Furthermore, one might want to note that, had (P) turned out to be a consequence of Strict Dominance, it would have followed that $(\mathrm{P})$ is after all satisfied by a large proportion of the aforementioned imprecise decision rules, since it is easy to show that, with the exception of InTERval Dominance, the latter all satisfy the stronger principle. ${ }^{19}$

So there is no obvious shortcut to justifying a commitment to (P). But providing such a justification is absolutely crucial since, as it turns out, it does not take much of a weakening to yield a principle that is demonstrably satisfied by at least one popular rule for decision-making with imprecise probabilities, namely $\Gamma$-Maximin. ${ }^{20}$

\footnotetext{
${ }^{17}$ Note that there is some disagreement over the appropriate notion of independence at play here. On the evidentialist construal that we have offered here, this is simply taken to be probabilistic independence: two partitions $\mathbb{A}$ and $\mathbb{E}$ are independent just in case, for all $\operatorname{Pr} \in \Gamma_{t}, A \in \mathbb{A}$ and $E \in \mathbb{E}, \operatorname{Pr}(A \cap E)=\operatorname{Pr}(A) \operatorname{Pr}(E)$.

${ }^{18}$ That Elga's case is set up so that our rational agent is not in a position to exercise such a choice is perfectly legitimate. Indeed, possession of the ability to 'bind' oneself from the outset to a future sequence of acts, convenient as this may sometimes be, is certainly not a requirement of rationality. This would amount to rationality's imposing demands on the contents of the alternative sets that are presented to the agent, contents that is patently none of rationality's business.

${ }^{19}$ See the Appendix for a proof. Note that Strict Dominance's stronger relative, Weak DomiNANCE, is however notoriously violated by both Г-MAXimin and Г-MAximax (Luce \& Raiffa 1989, p. 287). Furthermore, since these two proposals offer constraints on choice sets that are strictly more stringent than those offered by the remainder of the decision rules that we have considered, the latter will also violate this stronger principle. (Reminder: WEAK Dominance excludes from the choice set those acts that are merely weakly dominated, where weak dominance requires simply weak inequality of the expectations for all $E \in \mathbb{E}$ and strict inequality for at least one.) I personally find Weak Dominance to be a rather dubious principle. Many others, however, apparently do not. For those of these who are wedded to the imprecise view, there remains the somewhat ad hoc move to tack on a stipulation to the effect that choice sets exclude weakly dominated acts.

${ }^{20} \Gamma$-Maximin has most notably been endorsed by Gilboa \& Schmeidler (1989). It is not, however, entirely uncontentious. Seidenfeld (2004), in particular, criticises it on the basis that it can sometimes license a negative valuation of cost-free information.
} 
This principle, which supplements the antecedent of Elga's own offering with a set of fairly weak conditions regarding the agent's beliefs about her future self, is the following:

$\left(\mathbf{P}^{-}\right)$A rational agent will either choose $A_{1}$ or choose $B_{1}$, given (FD), (NC) and

Certainty of Future Rationality (CFR): For all functions in $\Gamma_{1}$, the probability assigned to the agent's making a rational choice at time 2 is 1 .

Introspection (I): For all functions in $\Gamma_{1}$, the probability assigned to the agent's credal set being $\Gamma_{1}$ at time 1 is 1 .

Certainty of No Change in Belief in $H(C N C)$ : For all functions in $\Gamma_{1}$, the probability assigned to there being a function $\operatorname{Pr}$ in $\Gamma_{2}$ such that $\operatorname{Pr}(H)=r$ is equal to 1 conditional on there being such a function in $\Gamma_{1}$, and equal to 0 conditional on there not being such a function in $\Gamma_{1}$.

Perfect Recall $(P R)$ For all functions in $\Gamma_{2}$, and $1 \leq i \leq 2$, if $A_{i}$ was chosen at time 1 , then the probability assigned to $A_{i}$ is 1 .

Certainty of Perfect Recall (CPR) For all functions in $\Gamma_{1}$, and $1 \leq i \leq$ 2 , the probability assigned to its being the case that, for all $\operatorname{Pr}$ in $\Gamma_{2}$, $\operatorname{Pr}\left(A_{i}\right)=1$, conditional on $A_{i}$, is 1 .

That $\Gamma$-Maximin does indeed satisfies this principle can now be established as follows:

Let $\Gamma_{1}$ be such that the probability accorded to $H$ lies between $a$ and $b$ : $\min \left\{\operatorname{Pr}(H): \operatorname{Pr} \in \Gamma_{1}\right\}=a$ and $\max \left\{\operatorname{Pr}(H): \operatorname{Pr} \in \Gamma_{1}\right\}=b$. It will be shown that, depending on the value of $a$, one or the other of the two following disjuncts hold: either choosing $A_{2}$ will mandate choosing $B_{1}$, or choosing $A_{1}$ will be mandatory in the first place.

Either (1) $a>2 / 5$ or (2) $a \leq 2 / 5$. Assume (1). Now, by (NC) and (PR), if $A_{2}$ were to be chosen at time 1 , according to $\Gamma_{2}$, a choice of $B_{1}$ at time 2 would have, at worst, an expected payoff of $15 a-10(1-a)=25 a-10,{ }^{21}$ whereas a choice of $B_{2}$ would have, at worst, an expected payoff of 0 utiles. Since (1) is equivalent to $25 a-10>0$, it follows from $\Gamma$-M chosen at time 1 , then $B_{1}$ would be chosen at time 2 . This is the first of our disjuncts.

So now assume (2), i.e. that $a \leq 2 / 5$. This is equivalent to $0 \geq 25 a-10$. Let $\operatorname{Pr}$ be one of the functions in $\Gamma_{1}$ that assigns a probability of $a$ to $H$. According to $\operatorname{Pr}, 25 a-10$ corresponds to the expected payoff of choosing $A_{2}$ and then $B_{1}$, and 0 corresponds to the expected payoff of choosing $A_{2}$ and then $B_{2}$.

\footnotetext{
${ }^{21}$ Since the expected payoff of $A_{2} \cap B_{1}$ is higher conditional on $H$ than it is on its negation, it is minimised by minimising the probability of $H$.
} 
Given (FD), the expected payoff of choosing $A_{2}$, according to $\mathrm{Pr}$, will be a weighted average of these two values and hence, by the previous inequality, no greater than 0 . So a fortiori, the lowest expected payoff of choosing $A_{2}$, with respect to $\Gamma_{1}$, will itself be no greater than 0 .

Now by (CPR), (I) and (CNC), it follows that (3): Every function in $\Gamma_{1}$ accords a probability of 1 , conditional on $A_{1}$ 's being chosen at time 1 , to the proposition that, according to $\Gamma_{2}$, a choice of $B_{2}$ at time 2 would have, at worst, an expected payoff of $-10 b+15(1-b)=15-25 b,{ }^{22}$ whereas a choice of $B_{1}$ would have, at worst, an expected payoff of 5 utiles.

We now have three cases to consider: (i) $15-25 b>5$, (ii) $15-25 b=5$ and (iii) $15-25 b<5$. Given (CFR) and $\Gamma$-Maximin, we recover the three following conditionals:

If (i), then, for every function in $\Gamma_{1}$, the probability assigned to choosing $B_{2}$ conditional on having chosen $A_{1}$ will be 1 . From this, it follows that, for every function, the expected value of choosing $A_{1}$ will be at least as great as $15-25 b$ (since the latter minimises this expected value) and thus, by (i) again, strictly greater than 5 .

If (ii), then, for every function in $\Gamma_{1}$, the probability assigned to choosing $B_{1}$ conditional on having chosen $A_{1}$ will be 1 . From this, it follows that, for every function, the expected value of choosing $A_{1}$ will be equal to 5 . If (iii), then, for every function in $\Gamma_{1}$, the expected value of choosing $A_{1}$ will be equal to 5 .

Whichever one of (i) to (iii) holds, the lowest expected payoff of choosing $A_{1}$ will be at least as great as 5 . Since, as we have seen, the lowest expected payoff of choosing $A_{2}$ will be no greater than $0, \Gamma$-MAXIMIN will mandate a choice of $A_{1}$. We now have the second disjunct. QED. ${ }^{23}$

Whether $\Gamma$-Maximax violates or satisfies $\left(\mathrm{P}^{-}\right)$remains an open question. However, $\left(\mathrm{P}^{-}\right)$certainly remains a strong enough principle to be violated by E-ADMISSIBILITY, Maximality and Interval Dominance. We establish the result for E-Admissibility. This then carries over to Maximality and Interval Dominance, since, as noted above in Section 3, the latter are strictly more permissive than the former. ${ }^{24}$

Consider $\Gamma_{1}=\left\{\operatorname{Pr}_{1}, \operatorname{Pr}_{1}^{*}\right\}$, such that $\operatorname{Pr}_{1}(H)=0$ and $\operatorname{Pr}_{1}^{*}(H)=1$. We first note that, given our assumptions, no constraint is imposed by E-AdmissibILity on

\footnotetext{
${ }^{22}$ Since the expected payoff of $A_{1} \cap B_{2}$ is lower conditional on $H$ than it is on its negation, it is minimised by maximising the probability of $H$.

${ }^{23}$ Note that this proof obviously also establishes that the sharp view satisfies the principle: simply let $a=b$.

${ }^{24}$ The fact that these rules lead to a violation of $\left(\mathrm{P}^{-}\right)$obviously entails that they also lead to a violation of Elga's strictly stronger $(\mathrm{P})$. The present result thus provides a basis for a fragment of his descriptive claim.
} 
what choice will be made at time 2 conditional on whatever choice was made at time 1 . To see why, assume for instance that $A_{2}$ was chosen at time 1 . By (PR), $\Gamma_{2}$ is obtained by conditionalising each of the two members of $\Gamma_{1}$ on $A_{2}$. Furthermore, since the probabilities of $H$ are extremal in both cases, they remain unchanged, at 0 and 1 , respectively. It then follows that, according to $\operatorname{Pr}_{1}$ 's counterpart in $\Gamma_{2}$-call it $\operatorname{Pr}_{2}$-the expected utility of $B_{2}$ is equal to 0 , which is strictly greater than -10 , the expected utility of $B_{1}$. According to $\operatorname{Pr}_{1}^{*}$ 's counterpart in $\Gamma_{2}$-call it $\operatorname{Pr}_{2}^{*}$-however, this inequality is reversed: an expected utility of 15 for $B_{1}$, against an expected utility of 0 for $B_{2}$. Thus E-Admissibility allows both $B_{1}$ and $B_{2}$ into the choice set. If one assumes instead that $A_{1}$ was chosen, we recover the same conclusion by an analogous chain of reasoning.

Given (I), (CFR) and (CPR) it then follows that E-AdmissibILITY imposes no constraints on the agent's beliefs about what choice will be made at time 2 conditional on whatever choice was made at time 1: $\operatorname{Pr}_{i}\left(B_{j} \mid A_{k} \cap H\right)$ and $\operatorname{Pr}_{i}\left(B_{j} \mid A_{k} \cap \bar{H}\right.$ ) (where $i, j, k \in\{1,2\}$ ) are unconstrained.

So let us assume that, say, $\operatorname{Pr}_{i}\left(B_{j} \mid A_{k} \cap H\right)=\operatorname{Pr}_{i}\left(B_{j} \mid A_{k} \cap \bar{H}\right)=0$.5. It follows from this that $A_{2}$ maximizes expected utility according to $\operatorname{Pr}_{1}$ and hence, by E-ADMissibiLITY, constitutes a permissible choice. Indeed:

$$
\begin{aligned}
\mathrm{E}_{\mathrm{Pr}_{1}}\left(\mathrm{U} \mid A_{1}\right) & =\mathrm{E}_{\mathrm{Pr}_{1}}\left(\mathrm{U} \mid A_{1} \cap \bar{H}\right) \\
& =\operatorname{Pr}_{1}\left(B_{1} \mid A_{1} \cap \bar{H}\right)(5)+\operatorname{Pr}_{1}\left(B_{2} \mid A_{1} \cap \bar{H}\right)(15) \\
& =10 \\
\mathrm{E}_{\mathrm{Pr}_{1}}\left(\mathrm{U} \mid A_{2}\right) & =\mathrm{E}_{\mathrm{Pr}_{1}\left(\mathrm{U} \mid A_{2} \cap \bar{H}\right)} \\
& =\operatorname{Pr}_{1}\left(B_{1} \mid A_{2} \cap \bar{H}\right)(-10)+\operatorname{Pr}_{1}\left(B_{2} \mid A_{2} \cap \bar{H}\right)(0) \\
& =-5
\end{aligned}
$$

Since we saw above that no constraint is imposed by E-ADMISSIBILI-TY on what choice will be made at time 2 conditional on whatever choice was made at time 1 , it follows that it is permissible for our agent to choose $B_{2}$ on the heels of $A_{2}$, thus declining both risky prospects. QED.

\section{Concluding remarks}

We have seen that Elga's Todeserklärung for the imprecise view was somewhat premature. Not only (i) had Elga not established that all promising incarnations of the framework, in contrast to their sharp counterpart, violate his putative normative principle $(\mathrm{P})$, but (ii) he had provided no reason for us to endorse $(\mathrm{P})$ over a weaker counterpart $\left(\mathrm{P}^{-}\right)$that is demonstrably satisfied by at least one popular variety of the imprecise view. 
But we have also seen that, quite interestingly, a number of well-known decision rules do not satisfy even $\left(\mathrm{P}^{-}\right)$. So, were it possible to make a convincing case for the latter, a task that I have not attempted to undertake here, this last observation would take us a step further towards resolving the internal dispute between competing factions within imprecise camp.

\section{Appendix}

Proof that the sharp view satisfies $(P)$ : Assume for reductio that the agent chooses both $A_{2}$ and $B_{2}$. It follows from the latter that

$$
\mathrm{E}_{\mathrm{Pr}_{2}}\left(\mathrm{U} \mid B_{2}\right)-\mathrm{E}_{\mathrm{Pr}_{2}}\left(\mathrm{U} \mid B_{1}\right) \geq 0
$$

For ease of notation, let us (i) denote by $\$\left(A_{i} \mid H\right)$ the payoff, in utiles, received upon having performed action $A_{i}$ in state $H$ and (ii) denote $H$ and $\bar{H}$ by $H_{1}$ and $H_{2}$ respectively. We note that

$$
\mathrm{E}_{\mathrm{Pr}_{2}}\left(\mathrm{U} \mid B_{j}\right)=\sum_{i, k}\left(\$\left(A_{i} \mid H_{k}\right)+\$\left(B_{j} \mid H_{k}\right)\right) \operatorname{Pr}_{2}\left(A_{i} \cap H_{k}\right)
$$

So we therefore have

$$
\begin{aligned}
\mathrm{E}_{\mathrm{Pr}_{2}}\left(\mathrm{U} \mid B_{2}\right)-\mathrm{E}_{\mathrm{Pr}_{2}}\left(\mathrm{U} \mid B_{1}\right) & =\sum_{i, k}\left(\$\left(B_{2} \mid H_{k}\right)-\$\left(B_{1} \mid H_{k}\right)\right) \operatorname{Pr}_{2}\left(A_{i} \cap H_{k}\right) \\
& =\sum_{i}(0-15) \operatorname{Pr}_{2}\left(A_{i} \cap H_{1}\right)+(0-(-10)) \operatorname{Pr}_{2}\left(A_{i} \cap H_{2}\right) \\
& =-15 \operatorname{Pr}_{2}\left(H_{1}\right)+10 \operatorname{Pr}_{2}\left(H_{2}\right)
\end{aligned}
$$

From (1) and (2), it then follows that $\operatorname{Pr}_{2}\left(H_{1}\right) \leq 2 / 5$, and from this, by (NC), that $\operatorname{Pr}_{1}\left(H_{1}\right) \leq 2 / 5$. But by a similar chain of reasoning, we can derive $\operatorname{Pr}_{1}\left(H_{1}\right)>2 / 5$ from the assumption that $A_{2}$ is chosen. Contradiction. QED.

Proof that Strict Dominance is satisfied by all imprecise decision rules considered, apart from InTERval Dominance: Where $A_{1}, A_{2} \in \mathbb{A}$, if $A_{1}$ strictly dominates $A_{2}$, then, for all $\operatorname{Pr} \in \Gamma, \mathrm{E}_{\mathrm{Pr}}\left(\mathrm{U} \mid A_{2}\right)>\mathrm{E}_{\mathrm{Pr}}\left(\mathrm{U} \mid A_{1}\right)$, so that, according to Maximality, $A_{2} \notin \mathrm{C}(\mathbb{A})$. Since $\Gamma$-Maximax, $\Gamma$-Maximin and E-Admissibility are all strictly more stringent than Maximality, they will deliver the same verdict.

Regarding Interval Dominance: Consider partitions $\mathbb{A}=\left\{A_{1}, A_{2}\right\}$ and $\mathbb{E}=$ $\left\{E_{1}, E_{2}\right\}$, with $\Gamma=\left\{\operatorname{Pr}, \operatorname{Pr}^{*}\right\}$, such that $\operatorname{Pr}\left(E_{1}\right)=1$ and $\operatorname{Pr}^{*}\left(E_{1}\right)=0$ and $\mathbb{A}$ and $\mathbb{E}$ are independent. Let the expectations of $\mathrm{U}$ conditional on $A_{1} \cap E_{1}, A_{1} \cap E_{2}, A_{2} \cap E_{1}$ and $A_{2} \cap E_{2}$ be $2,4,1$ and 3 , respectively, so that $A_{1}$ strictly dominates $A_{2}$. It follows however that $\max \left\{\mathrm{E}_{\mathrm{Pr}}\left(\mathrm{U} \mid A_{2}\right): \operatorname{Pr} \in \Gamma\right\}=3>2=\min \left\{\mathrm{E}_{\mathrm{Pr}}\left(\mathrm{U} \mid A_{1}\right): \operatorname{Pr} \in \Gamma\right\}$, so that, according to Interval Dominance, $A_{2} \in \mathrm{C}(\mathbb{A})$. QED. 


\section{Acknowledgments}

The research for this article was supported by a Research Foundation-Flanders (FWO) postdoctoral research grant and facilitated by the European Rationality $\&$ Decisions Network, funded by the Netherlands Organisation for Scientific Research (NWO) (grant nr. 236-20-005). I am grateful to Richard Bradley, Igor Douven and Wlodek Rabinowicz for helpful feedback on an early version of this paper. I would also like to thank the audiences of the MCMP Colloquium in Logic, Philosophy of Science and Philosophy (LMU Munich, 2011), the KU Leuven Public Economics seminar (KU Leuven, 2011), the Bayreuth Philosophy \& Economics Research Colloquium (Bayreuth, 2011) and the Rationality \& Decisions Network workshop on Radical Uncertainty (LMU Munich, 2012) for their comments.

\section{References}

Camerer, C. and M. Weber (1992). Recent developments in modeling preferences: Uncertainty and ambiguity. Journal of Risk and Uncertainty 5:325-370.

Ellsberg, D. (1961). Risk, ambiguity, and the Savage axioms. Quarterly Journal of Economics 75(4):643-669.

Elga, A. (2010). Subjective probabilities should be sharp. Philosopher's Imprint, 10(5), pp. 1-11.

Jeffrey, R. (1983). The Logic of Decision. Second edition. Chicago: University of Chicago Press.

Jeffrey, R. (1987). Indefinite probability judgment: A reply to Levi. Philosophy of Science 54(4):586-591.

Joyce, J. (1999). The Foundations of Causal Decision Theory. Cambridge: Cambridge University Press.

Joyce, J. (2010). A defense of imprecise credences in inference and decision making. Philosophical Perspectives 24: 281-323.

Levi, I. (1980). The Enterprise of Knowledge: An Essay on Knowledge, Credal Probability and Chance. Camb. Mass.: MIT Press.

Luce, R. D. \& Raiffa, H. (1989). Games and Decisions: Introduction and Critical Survey. Dover Publications.

Gilboa, I. and D. Schmeidler (1989). Maxmin expected utility with non-unique prior. Journal of Mathematical Economics, 18, pp. 141-53. 
Machina, M.J. (1987). Choice under uncertainty: Problems solved and unsolved. The Journal of Economic Perspectives, 1(1):121-154.

Miranda, E. (2008). A survey of the theory of coherent lower previsions. International Journal of Approximate Reasoning, 48(2), pp. 628-58.

Seidenfeld, T. (2004). A contrast between two decision rules for use with (convex) sets of probabilities: $\gamma$-Maximin versus E-Admissibility. Synthèse, 140(1/2), pp. 69-88.

Seidenfeld, T. \& L. Wasserman, L. (1993). Dilation for sets of probabilities. The Annals of Statistics, 21:1139-1154.

Shackle, N. (2007). Bertrand's Paradox and the Principle of Indifference. Philosophy of Science 74:150-175.

Troffaes, M. C. M. (2007). Decision making under uncertainty using imprecise probabilities. International Journal of Approximate Reasoning, 45, pp. 17-29.

van Fraassen, B. (1989). Laws and Symmetry. Oxford: OUP.

Walley, P. (1991). Statistical Reasoning with Imprecise Probabilities. London: Chapman and Hall.

Weirich, P. (2008). Causal decision theory. In E. Zalta (ed.) Stanford Encyclopaedia of Philosophy.

White, R. (2010). Evidential symmetry and mushy credence. In Gendler, T.S. and Hawthorne, J. (eds.), Oxford Studies in Epistemology, vol. 3, pp. 161-186. Oxford University Press. 